\title{
Best Constant in Sobolev Inequality (*).
}

\author{
GroRgto TALENTI (Firenze)
}

Summary. - The best constant for the simplest Sobolev inequality is exhibited. The proof is accomplished by symmetrizations (rearrangements in the sense of Hardy-Littlewood) and onedimensional calculus of variations.

0. - The main result of the present paper is the following:

THEOREM. - Let $u$ be any real (or complex) valued function, defined on the whole m-dimensional euclidean space $R^{m}$, sufficiently smooth and decaying fast enough at infinity. Moreover let $p$ be any number such that: $1<p<m$. Then:

$$
\left\{\int_{R^{m}}|u|^{\mid \alpha} d x\right\}^{1 / \alpha} \leqslant C\left\{\int_{R^{m}}|D u|^{p} d x\right\}^{1 / s}
$$

where: $|D u|$ is the length of the gradient $D u$ of $u, q=m p /(m-p)$ and

$$
C=\pi^{-\frac{1}{2}} m^{-1 / p}\left(\frac{p-1}{m-p}\right)^{1-1 / p}\left\{\frac{\Gamma(1+m / 2) \Gamma(m)}{\Gamma(m / p) \Gamma(1+m-m / p)}\right\}^{1 / m}
$$

The equality sign holds in (1) if u has the form:

$$
u(x)=\left[a+b|x|^{p /(p-1)}\right]^{1-m / p},
$$

where $|x|=\left(x_{1}^{2}+\ldots+x_{m}^{2}\right)^{\frac{1}{2}}$ and $a, b$ are positive constants.

Sobolev inequalities, also called Sobolev imbedding theorems, are very popular among writers in partial differential equations or in the calculus of variations, and have been investigated by a great number of authors. Nevertheless there is a question concerning Sobolev inequalities, which seems well-known only to a restricted number of specialists working in geometric measure theory. The question is the connection between Sobolev inequalities and the classical isoperimetric inequality for subsets of euclidean spaces. Our aim is to advertise such a connection.

To be specific, we are concerned with the simplest Sobolev inequality

$$
\|u\|_{L^{a}\left(R^{m}\right)} \leqslant(\text { constant independent of } u)\|D u\|_{L^{p}\left(R^{m}\right)},
$$

(*) Entrata in Redazione il 16 luglio 1975 . 
and we are interested in the smallest constant which is admissible in (4); namely we will evaluate the expression

$$
C=\sup \frac{\|u\|_{L^{q}\left(R^{m}\right)}}{\|D u\|_{L^{p}\left(R^{m}\right)}}
$$

where the sup is taken in the class of all (not identically zero) smooth (e.g. Lipschitz continuous) functions $u$ which decay rapidly at infinity (e.g. compactly supported) Easy tests and arguments of dimensional analysis show that (4) is impossible (equivalently $C=+\infty$ ) if $p \geqslant m$ or $q \neq m p /(m-p)$. Incidentally, dimensional analysis shows also that the supremum in question does not change if the competiting functions are restricted to have their support in some fixed open set.

If $1<p<m$ and $q$ has the value $m p /(m-p),(4)$ can be proved for functions $u$ in $C_{0}^{\mathrm{J}}\left(R^{m}\right)$ (i.e. continuously differentiable functions with compact support) by using the straightforward representation formula

$$
u(x)=-\frac{\Gamma(m / 2)}{2 \pi^{m / 2}} \int_{R^{m}}|x-y|^{1-m} \sum_{k=1}^{m} \frac{x_{k}-y_{k}}{|x-y|} \frac{\partial u}{\partial x_{k s}}(y) d y
$$

and by applying to the right-hand side an $m$-dimensional version of a theorem of Hardy-Littlewood concerning fractional integrals. This is the method of SoboLev [11-12]; for a concise presentation see Bers-JoHN-Schechter [1]. Unfortunately, the Sobolev method neither gives the exact value of the best constant $C$ nor explicit estimates for $O$.

The theorem we present in this paper gives the sharp form of the inequality (4). It turns out that the best constant $O$ of the Sobolev inequality has the value (2). Moreover the ratio between the $L^{q}$-norm of $u$ and the $L^{p}$-norm of the gradient of $u$ attains its maximum value $C$ on functions $u$ of the form (3).

We notice that a discussion of the sharp form of Sobolev inequality, restricted to the case $m=3, p=2, q=6$, is in Rosen [10].

We emphasize that our result is valid only if $p \neq 1$. If $p=1$, the Sobolev inequality behaves in a slightly different manner. In fact, it must be expected that the ratio between a norm of $u$ and the integral $\int|D u| d x$ attains its maximum value on functions $u$ whose gradient is a (not absolutely continuous) measure. For instance, if we look at the trivial one-dimensional inequality

$$
\left\{\int_{-\infty}^{+\infty}|u|^{\alpha} d x\right\}^{1 / \alpha} \leqslant \frac{L^{1 / \alpha}}{2} \int_{-\infty}^{+\infty}\left|u^{\prime}\right| d x, \quad \text { support of } u \subset[0, L]
$$

we see at once that all functions for which the inequality becomes an equality are step functions equal to some constant in the interior of the interval $[0, L]$ and vanishing outside: of course for functions with jump discontinuities the integral 
$\int\left|u^{\prime}\right| d x$ must be interpreted as the total variation of $u$. Accordingly, it turns out that functions maximizing the ratio between the two sides of Sobolev inequality are characteristic functions of balls when $p=1$. This is essentially a result of FEDERERFlemivg [4] and Fleming-RIshei [6]. Let us describe briefly the situation.

Federer, Fleming and Rishei proved the inequality:

$$
\left\{\int_{R^{m}}|u|^{m /(m-1)} d x\right\}^{1-1 / m} \leqslant \frac{\{\Gamma(1+m / 2)\}^{1 / m}}{\sqrt{\bar{\pi}} m} \int_{R^{m}}|D u| d x
$$

for every $u$ belonging to a broad class of functions which vanish at infinity. Note that the constant appearing in (5) is the limiting value (as $p \rightarrow 1$ ) of the rightside of (2). The inequality (5) is sharp, for an easy computation shows:

$\left\{\int_{R^{m}}\left|u_{n}\right|^{m /(m-1)} d x\right\}^{1-1 / m}\left\{\int_{R^{m}}\left|D u_{n}\right| d x\right\}^{-1}=\frac{\{\Gamma(1+m / 2)\}^{1 / m}}{\sqrt{\pi} m}[1+O(1 / n)] \quad($ if $n \rightarrow+\infty)$,

where $u_{n}(x)=1$ if $0 \leqslant|x| \leqslant 1, u_{n}(x)=1+n-n|x|$ if $1<|x|<1+1 / n$ and $u_{n}(x)=0$ if $|x| \geqslant 1+1 / n$. This is a sequence of Lipschitz continuous functions which converges to the characteristic function of the unit ball.

The constant in the right-hand side of $(5)$ is the isoperimetric constant; i.e. the constant which appears in the classical isoperimetric inequality

$$
\left\{H_{m}(E)\right\}^{1-1 / m b} \leq \frac{\{\Gamma(1+m / 2)\}^{1 / m}}{\sqrt{ } \bar{\pi} m} H_{m-1}(\partial E),
$$

where

$$
\begin{aligned}
& E=\text { any bounded smooth subset of } R^{m} ; \\
& \partial E=\text { the boundary of } E \\
& H_{k}=\text { the } k \text {-dimensional (Hausdorff) measure. }
\end{aligned}
$$

There is a deep connection between the Federer-Fleming-Rishel version of the Sobolev inequality and the isoperimetric inequality. Indeed Federer, Fleming and RIsHex deduce their inequality from the isoperimetric inequality. On the other hand, it is easy to derive the isoperimetric inequality from the Federer-FlemingRishel result. Let us sketch such a derivation.

The key to the derivation is a suitable definition of the total variation for funetions of several real variables. The total variation of a real valued locally integrable function $u$ of one real variable is

$$
\sup \left\{\sum_{k=1}^{N}\left|u\left(x_{k}\right)-u\left(x_{i-1}\right)\right|:-\infty<x_{1}<x_{\mathbf{z}}<\ldots<x_{N}<+\infty\right\}
$$


it is well-known and easy to see that this is the smallest constant $C$ such that

$$
\left|\int_{-\infty}^{+\infty} u(x) \varphi^{\prime}(x) d x\right| \leqslant C \max |\varphi(x)|
$$

for every $\varphi$ in $C_{0}^{1}(]-\infty,+\infty[$ ). If $u$ is smooth (e.g. absolutely continuous), then the total variation of $u$ equals $\int_{-\infty}^{+\infty}\left|u^{\prime}(x)\right| d x$. Accordingly, we define the total variation of a real valued locally integrable function $u$, defined over $R^{m}$, as

$$
\sup \left\{\int_{R^{m}} u \sum_{k=1}^{m} \frac{\partial v_{k}}{\partial x_{k}} d x: v_{k} \in O_{0}^{1}\left(R^{m}\right), \max \sum_{k=1}^{m} v_{k}(x)^{2} \leqslant 1\right\} .
$$

Clearly

$$
\text { total var. of } u=\int_{R^{m}}|D u| d x
$$

whenever $u$ is continuously differentiable.

By the way, the set of all functions which are integrable in $R^{m}$ and whose total variation is finite is usually called $B V\left(R^{m}\right)$; see e.g. [5], [7].

The inequality (5) tell us that

$$
\left\{\int_{R^{m}}|u|^{\mid m m /(m-1)} d x\right\}^{1-1 / m} \leqslant \frac{\{\Gamma(1+m / 2)\}^{1 / m}}{\sqrt{\pi} m} \times \text { total var. of } u,
$$

for every function $u$ in $B V\left(R^{m}\right)$. This is obvious if $u$ is continuously differentiable. If $u$ is not continuously differentiable, (6) can be deduced from (5) using the customary mollifiers technique and the easy fact that mollifications do not increase the total variation. It should be noticed that the full result of FEDERER, Fuentrg and RIsHeL is precisely the inequality (6).

Now it is quite clear that (6) contains the isoperimetric inequality as a particular case. Let $E$ be a bounded smooth subset of $R^{m}$, and apply (6) to $1_{B}$, the eharacteristic function of $E$.

The left hand side then becomes the $(1-1 / m)$-th power of the measure of $E$, while on the right hand side we have that the

$$
\text { total variation of } \mathbf{1}_{E}=\sup \left\{\int_{\partial E} \sum_{k=1}^{m} v_{k}(x) X_{k}(x) H_{m-1}(d x): \max \sum_{k=1}^{m} v_{k}(x)^{2} \leqslant 1\right\},
$$

where $X$ is the unit normal vector to $\partial E$; this follows from the Gauss-Green formulas, Hence:

total variation of $\mathbf{1}_{E}=$ the $(m-1)$-dimensional measure of the boundary of $\mathbb{E}$. 
We mention that a connection between a Sobolev inequality and an isoperimetric inequality for point sets can be established also if the euclidean space $R^{m}$ is replaced by special non-flat manifolds; see M. MIranda [9].

Of course we have assumed in the previous discussion that $m$, the dimension of the ground space, is greater than one. In one dimension, an analogue of the Sobolev inequality is perhaps the following:

$$
\left[\int_{0}^{L}|u|^{q} d x\right]^{1 / q} \leqslant L^{1 / q+1 / p^{\prime}} \frac{q\left(1+p^{\prime} / q\right)^{1 / p}}{2\left(1+q / p^{\prime}\right)^{1 / \alpha} B\left(1 / q, 1 / p^{\prime}\right)}\left[\int_{0}^{L}\left|u^{\prime}\right|^{p} d x\right]^{1 / p}
$$

where $u$ is any real valued absolutely continuous function on a bounded interval $[0, L]$ with

$$
u(0)=u(L)=0
$$

and $p, p^{\prime}, q$ are numbers such that $1 \leqslant q<\infty, 1<p<\infty, p^{\prime}=p /(p-1)$. The constant in (7a) is the best; in fact, equality holds in (7a) if and only if $u$ is the function implicitely defined by the relations

$$
\left\{\begin{array}{l}
\int_{0}^{u(x)}\left(1-t^{2}\right)^{-1 / p} d t=\frac{\Gamma\left(1 / p^{\prime}\right) \Gamma(1+1 / q)}{\Gamma\left(1 / p^{\prime}+1 / q\right)} \frac{2 x}{L} \quad \text { if } 0 \leqslant x \leqslant \frac{L}{2} \\
u(x)=u(L-x),
\end{array}\right.
$$

or if $u$ is a numerical multiple of such a function. The inequality (7) might be proved with standard techniques of the calculus of variations.

\section{1. - Symmetrization.}

The proof of the theorem stated at the beginning consists of two steps. In the first step we prove that the ratio

$$
\frac{\|u\|_{L^{g}\left(R^{m}\right)}}{\|D u\|_{L^{p}\left(R^{m}\right)}}
$$

attains its maximum value on spherically symmetrie functions. In the second step we prove that the ratio (8) actually has a maximum in a class of spherically symmetric functions; moreover we prove that such a maximum has the value (2), the maximizing spherically symmetric functions being of the form (3).

Of course, if $u$ is a Lipschitz function, then the ratio (8) is not altered whenever we replace $u$ by $|u|$. Hence in estimating the sup of (8) we can cut of the competition all functions which change their algebraic sign. 
To be more precise, in the first step we prove that the functional (8) increases if $u$ is replaced by a suitable rearrangement of $u$; namely by a function $u^{*}$ whose level sets $\left\{x \in R^{m}: u^{*}(x)>t\right\}$ are balls which have the same measure as the level sets $\left\{x \in R^{m}: u(x)>t\right\}$ of $u\left({ }^{1}\right)$. This statement is a consequence of the isoperimetric inequality for subsets of $R^{m}$, as the following lemma shows.

Lemma 1. - Let u be any smooth (e.g. Lipschitz continuous) real valued function defined on the whole euclidean space $R^{m}$ which decays fast enough at infinity (e.g. with compact support). Let $u^{*}$ be the spherically symmetric rearrangement of $u$; that is

$$
u^{*}(x)=\sup \left\{t: \mu(t)>C_{m}|x|^{m}\right\}
$$

where $C_{m}=\pi^{m / 2} / \Gamma(1+m / 2)$ is the measure of the m-dimensional unit ball and

$$
\mu(t)=\operatorname{meas}\left\{x \in R^{m}: u(x)>t\right\} .
$$

Moreover suppose that $u$ is nonnegative. Then, for every exponent $p \geqslant 1$, the following holds:

(i) $\int\left(u^{*}\right)^{p} d x=\int u^{p} d x$;

(ii) $\int\left[\left.D u^{*}\right|^{p} d x \leqslant \int|D u|^{p} d x\right.$.

Here and below, $\int \ldots d x$ stands for integral over the whole space $R^{m}$.

The property (i) is trivial since the integral of any power of a function is known when the measure of each level set is known.

This follows from the familiar formula (Bonaventura Cavalieri principle!):

$$
\int u^{p} d x=\int_{0}^{+\infty} \mu(t) d\left(t^{p}\right) .
$$

Once we observe that the level sets of $u^{*}$ are balls of radius $\left(\mu(t) / C_{m}\right)^{1 / m}$; i.e.

$$
\left\{x \in R^{m}: u^{*}(x)>t\right\}=\left\{x \in R^{m}:|x|<\left(\mu(t) / O_{m}\right)^{1 / m}\right\}
$$

and:

$$
\operatorname{meas}\left\{x \in R^{m}: u^{*}(t)>t\right\}=\mu(t)
$$

(1) The monotonocity of Rayleigh-type quotients under this rearrangement is a wellknown principle of Pólya and Szegö. It has been used by a great number of authors in the proof of isoperimetric inequalities, in estimates of eigenvalues, and in several other problems of analysis or differential geometry. See G. PóxyA - G. SzEGö, Isoperimelric inequalities in Mathematical Physics, Annals of Math. Studies 27, Princeton (1951); L. E. Payne, Isoperimetric inequalities and their applications, SIAM Review, 9 (1967).

Here we revisit a proof of the Pólya and Szegö principle. We are indebted to Prof. E. on GIORGI for helpful suggestions. 
The formula (12) is an easy consequence of the definition (9) since $\mu$ is a decreasing and right-continuous function of $t$. Incidentally, $\mu(t)=+\infty$ if $t<0$, $\mu(0)=$ meas (support of $u$ ) since $u$ is nonnegative and $\mu(t)$ vanishes if $t \geqslant \max u$. Moreover $\mu(t)$ decreases strictly from meas(sprt. $u$ ) to 0 in the interval $0 \leqslant t \leqslant \max u$ because, from the continuity of $u, \mu\left(t^{\prime}\right)-\mu\left(t^{\prime \prime}\right)=\operatorname{meas}\left\{x \in R^{m}: t^{\prime}<u(x) \leqslant t^{\prime \prime}\right\} \geqslant$ $\geqslant$ meas $\left\{x \in R^{m}: t^{\prime}<u(x)<t^{\prime \prime}\right\}=$ the measure of a non empty open set whenever $0 \leqslant t^{\prime}<t^{\prime \prime} \leqslant \max u$. Let us observe that $\mu$ will be actually discontinuous at every value of $t$ which is attained by $u$ on a set of positive measure; indeed $\mu(t-)-\mu(t)=$ $=\operatorname{meas}\left\{x \in R^{m}: u(x)=t\right\}$.

For the proof of (ii) we need results from the theory of functions of several real variables which are, in some sense, companions of the formula (11). Formula (11) expresses the integral of $u$ (or of a power of $u$ ) by means of the measures $\mu(t)$ of the level sets of $u$. There are formulas connecting the integral of $|D u|$ (i.e. the total variation of $u)$ with the $(m-1)$-dimensional measures of the boundaries, $\partial\left\{x \in R^{m}: u(x)>t\right\}$, of the level sets of $u$, or with the $(m-1)$-dimensional measures of the level surfaces $\left\{x \in R^{m}: u(x)=t\right\}$. These formulas are due to Federer [3], Fueming-Rished [6], L. C. Young [13]; see also M. Mrranda [8]. The formula we shall use is that of Federer, which, in our case, reads

$$
\int|D u(x)| d x=\int_{0}^{+\infty} H_{m-1}\left\{x \in R^{m}: u(x)=t\right\} d t
$$

where $H_{m-1}$ stands for $(m-1)$-dimensional (Hausdorff) measure. A more general version of (14) (see [3]) is

$$
\int f(x)|D u(x)| d x=\int_{0}^{+\infty} d t \int_{u(x)=\ell} f(x) H_{m-1}(d x)
$$

where $f$ is any real valued integrable function. If $u$ were a signed function, the integrals at the right of $(14)$ and $(15)$ should be replaced by $\int_{-\infty}^{+\infty} \ldots d t$.
A rough proof of $(15)$ might be as follows. Let

$$
\varphi(t)=\int_{u(x)>t} f(x)|D u(x)| d x
$$

Clearly $\varphi(t)$ is a function of $t$ with bounded variation; in fact $\varphi(t)$ is monotone decreasing if $f$ is positive. The derivative of $\varphi(t)$ is readily seen to be

$$
\varphi^{\prime}(t)=-\int_{v(x)=\ell} f(x) H_{m-1}(d x)
$$

at every $t$ such that: $a$ ) the level surface $\left\{x \in R^{m}: u(x)=t\right\}$ contains no critical point of $u ; b) f$ is continuous on this level surface; c) $u$ is twice continuously dif- 
ferentiable in a neighbourhood of this level surface. In fact, if $t$ satisfies the above conditions and if $h$ is sufficiently close to zero, then we can describe the interspace $\left\{x \in R^{m}: t \leqslant u(x) \leqslant t+h\right\}$ with a system of curvilinear coordinates $[0, h] \times R^{m-1} \ni$ $\exists(\lambda, \xi) \rightarrow x(\lambda, \xi) \in R^{m}$ such that $x(\lambda, \cdot)$ is a parametric representation of the level surface $\left\{x \in R^{m}: u(x)=t+\lambda\right\}$ and the $x(\cdot, \xi)$ are parametric representations of orthogonal trajectories of the level surfaces of $u$. Such a coordinate system can be defined as follows: $x(\cdot, \xi)$ is a solution of the system of ordinary differential equations $d x / d \lambda=D u(x) /|D u(x)|^{2}, x(0, \cdot)$ is a parametric representation of the level surface $\left\{x \in R^{m}: u(x)=t\right\}$. From the equations $|\partial x / \partial \lambda|=1 /|D u|, \partial x / \partial \lambda$ is orthogonal to $\partial x / \partial \xi_{k},\left(\partial x / \partial \xi_{k}\right)(\lambda, \xi)=\left(\partial x / \partial \xi_{k}\right)(0, \xi)+0(\lambda)$, we see that the jacobian of the transformation is given by

$$
\frac{1}{|D u|}\left\{\sqrt{\operatorname{det}\left(g_{i k}\right)}+O(\lambda)\right\}
$$

where $g_{i k}$ is the inner product between $\left(\partial x / \partial \xi_{i}\right)(0, \xi)$ and $\left(\partial x / \partial \xi_{k}\right)(0, \xi)$, i.e. a coefficient of the riemannian metric on the surface $\left\{x \in R^{m}: u(x)=t\right\}$. Thus we obtain

$$
\begin{aligned}
\varphi(t+h)-\varphi(t) & =-\int_{0}^{h} d \lambda \int_{R^{m-1}} f(x(\lambda, \xi))\left\{\sqrt{\operatorname{det}\left(g_{i k}\right)}+O(\lambda)\right\} d \xi \\
& =-h \int_{u(x)=t} f(x) H_{m-1}(d x)+o(h) .
\end{aligned}
$$

Suppose now we are able to check that $\varphi(t)$ is piecewise absolutely continuous (i.e. the difference between $\varphi(t)$, and the piecewise constant function which has the same jumps as $\varphi(t)$, is absolutely continuous); this point is essential of course but we do not want to discuss it here. Then

$$
\varphi(+\infty)-\varphi(0-)=\int_{0}^{+\infty} \varphi^{\prime}(t) d t+\sum(\mathrm{jumps}) ;
$$

this is the same thing as (15). In faet $\varphi(+\infty)-\varphi(0-)=-\int f(x)|D u(x)| d x$, since $u$ is nonnegative; furthermore all jumps of $\varphi(t)$ vanish, since

$$
\varphi(t+)-\varphi(t-)=-\int_{u(x)=t} f(x)|D u(x)| d x
$$

and this integral is zero either because the $m$-dimensional measure of $\left\{x \in R^{m}\right.$ : $u(x)=t\}$ is zero or because $D u$ vanishes on this same set (according to a theorem of C. B. MoRrey).

Incidentally, let us state explicitely some properties of the level sets we have used. The $\partial\left\{x \in R^{m}: u(x)>t\right\}$ is a subset of $\left\{x \in R^{m}: u(x)=t\right\}$ because of the con- 
tinuity of $u$; more precisely, $\left\{x \in R^{m}: u(x)=t\right\}$ is the union of the disjoint sets consisting of the $\partial\left\{x \in R^{m}: u(x)>t\right\}$ and the set of all points interior to $\left\{x \in R^{m}: u(x) \leqslant t\right\}$ at which $u$ reaches the value $t$. The latter set is a collection of local maxima, so the gradient of $u$ vanishes identically in this set. Hence $\partial\left\{x \in R^{m}: u(x)>t\right\}$ agrees with $\left\{x \in R^{m}: u(x)=t\right\}$ if the latter is a regular surface, i.e. does not contain critical points of $u$. Note that if $u$ is very smooth, the set of all levels $t$ for which $\left\{x \in R^{m}: u(x)=t\right\}$ contains critical points of $u$ has (one-dimensional) measure zero, via Sard's theorem.

From the previous argument we deduce also the following form of the derivative of the measure of level sets of $u$ :

$$
\mu^{\prime}(t)=-\int_{u(x)=t} \frac{H_{m-1}(d x)}{|D u(x)|}
$$

at every $t$ such that no critical point of $u$ is on the level surface $\left\{x \in R^{m}: u(x)=t\right\}$, as long as $u$ is sufficiently smooth in a neighbourhood of this surface. Of course $\mu(t)$ is not differentiable at any value $t$ which is attained by $u$ on a set of positive measure or at critical points of $u$. If $u$ is infinitely differentiable, (16) holds at almost every $t$, via Sard's theorem.

Now we are in position to prove the assertion (ii) of the lemma. The following inequality holds at almost every $t$ :

$$
\int_{u(x)=t}|D u|^{p-1} H_{m-1}(d x) \geqslant\left[-\mu^{\prime}(t)\right]^{1-p}\left[H_{m-1}\left\{x \in R^{m}: u(x)=t\right\}\right]^{p} .
$$

If $p=1,(18)$ is trivial. If $p>1$, we obtain by Holder's inequality that

$$
\begin{aligned}
H_{m-1}\left\{x \in R^{m}: u(x)=t\right\}=\int_{u(x)=t}|D u|^{1-1 / p} \frac{1}{|D u|^{1-1 / p}} H_{m-1}(d x) \leqslant & \left\{\int_{u(x)=t}|D u|^{p-1} H_{m-1}(d x)\right\}^{1 / p}\left\{\int_{u(x)=t} \frac{H_{m-1}(d x)}{|D u|}\right\}^{1-1 / p}
\end{aligned}
$$

and then we apply the formula (16). It must be pointed out that inequality (18) can be proved without any reference to an explicit expression for the derivative $\mu^{\prime}(t)$. In fact from Holder's inequality we obtain

$$
(1 / h) \int_{t<u(x) \leqslant t+h}|D u| d x \leqslant\left\{\frac{1}{h} \int_{t<u(x) \leqslant t+h}|D u|^{p} d x\right\}^{1 / p}\left\{-\frac{\mu(t+h)-\mu(t)}{h}\right\}^{1-1 / p}
$$

and consequently

$$
-\frac{d}{d t} \int_{u(x)>t}|D u| d x \leqslant\left[-\mu^{\prime}(t)\right]^{1-1 / p}\left[-\frac{d}{d t} \int_{u(x)>t}|D u|^{p} d x\right]^{1 / p}
$$


at almost every $t$. On the other hand, from (15), we get

$$
\int_{u(x)>t}|D u| d x=\int_{i}^{+\infty} H_{m-1}\left\{x \in R^{m}: u(x)=t^{\prime}\right\} d t^{\prime}
$$

Hence

$$
-\frac{d}{d t} \int_{v(x)>t}|D u| d x=H_{m-1}\left\{x \in R^{m}: u(x)=t\right\}
$$

Analogously

$$
-\frac{d}{d t} \int_{u(x)>t}|D u|^{p} d x=\int_{u(x)>t}|D u|^{p-1} H_{m-1}(d x)
$$

at almost every $t$. Thus (18) is proved.

The isoperimetric inequality tells us that

$$
[\mu(t)]^{1-1 / m} \leqslant \frac{[\Gamma(1+m / 2)]^{1 / m}}{\sqrt{\bar{\pi} m}} H_{m-1}\left\{x \in R^{m}: u(x)=t\right\} .
$$

Now, from (15) we have the identity

$$
\int|D u|^{p} d x=\int_{0}^{+\infty} d t \int_{u(x)=t}|D u|^{p-1} H_{m-1}(d x)
$$

Therefore, from (18), (19) and (20), we obtain the estimate

$$
\int|D u|^{p} d x \geqslant(\sqrt{\pi} m)^{p}\{\Gamma(1+m / 2)\}^{-p / m} \int_{0}^{+\infty} \mu(t)^{p(1-1 / m)}\left|\mu^{\prime}(t)\right|^{1-p} d t .
$$

Clearly, the inequality (21) becomes an equality if $u$ is spherically symmetric. Indeed, the equality holds in (19) if the level set $\left\{x \in R^{m}: u(x)>t\right\}$ is a ball. The proof of (18) shows that equality holds in (18) if $|D u|$ is constant on the level surface $\left\{x \in R^{m}: u(x)=t\right\}$. Since the rearrangement of $u$, we have called $u^{*},(a)$ is spherically symmetric $(b)$ has level sets with the same measure $\mu(t)$ as the level sets of $u(c)$ is Lipschitz continuous; then we can write

$$
\int\left|\boldsymbol{D} u^{*}\right|^{p} d x=(\sqrt{\pi} m)^{p}[\Gamma(1+m / 2)]^{-p / m} \int_{0}^{+\infty} \mu(t)^{p(1-1 / m)}\left|\boldsymbol{\mu}^{\prime}(t)\right|^{1-p} d t
$$

From (21) and (22) the desired conclusion follows. 
It remains to prove (c). From the formula (15) we get

$$
\int_{t \geqslant u(x)>t-h}|D u(x)| d x=\int_{t=h}^{t} H_{m-1}\left\{x \in R^{m}: u(x)=t^{\prime}\right\} d t^{\prime}
$$

Then, using the isoperimetric inequality (19) and the monotonicity of $\mu$, we have:

$$
L(\mu(t-h)-\mu(t)) \geqslant \frac{\sqrt{\pi} m}{[\Gamma(1+m / 2)]^{1 / m}} \mu(t)^{1-1 / m} h
$$

for all $t$ and $h$ such that $t>h>0$. In (23) $L$ is the Lipschitz constant of $u$, i.e.

$$
L=\max |D u(x)|
$$

As is easy to see, the estimate (23) and the definition (9) of $u^{*}$ give

$$
\left|u^{*}(x)-u^{*}(y)\right| \leqslant L|| x|-| y||
$$

Lemma 1 is proved.

\section{2. - Spherically symmetric functions.}

If $u$ depends on $r=|x|$ only, the ratio (8) becomes

$$
2^{-1 / m} \pi^{-\frac{1}{2}}[\Gamma(m / 2)]^{1 / m} J(u)
$$

Where

$$
J(u)=\frac{\left(\int_{0}^{+\infty} r^{m-1}|u(r)|^{\alpha} d r\right)^{1 / q}}{\left(\int_{0}^{+\infty} r^{m-1}\left|u^{\prime}(r)\right|^{p} d r\right)^{1 / p}}
$$

LEMMa 2. - Let $m, p, q$ be real numbers such that

$$
1<p<m, \quad q=m p /(m-p)
$$

Let $u$ be any real valued funotion of a real variable $r$, which is sufficiently smooth on the half-line ]0, $+\infty$ [ (e.g. Lipschitz continuous) and which is such that

$$
\int_{0}^{+\infty} r^{m-1}\left|u^{\prime}(r)\right|^{p} d r<+\infty, \quad u(r) \rightarrow 0 \text { if } r \rightarrow+\infty
$$


Then

$$
J(u) \leqslant J(\varphi)
$$

where $\varphi$ is any function of the form

$$
\varphi(r)=\left(a+b r^{p^{\prime}}\right)^{1-n / p}
$$

with $a$ and $b$ positive constants.

An easy computation gives

$$
J(\varphi)=m^{-1 / p}\left(\frac{p-1}{m-p}\right)^{1 / p^{\prime}}\left[\frac{1}{p^{\prime}} B\left(m / p, m / p^{\prime}\right)\right]^{-1 / m} .
$$

Here $p^{i}=p /(p-1)$.

The lemma 2 exhibits the maximum value and maximizing functions of the functional $J$ as given by (24). The proof will be modelled on the classical patterns of the calculus of variations; we shall use Mayer fields of extemals, the Hilbert invariant integral, and the Weierstrass $E$ function ( ${ }^{2}$ ).

The domain we assign to the functional $J$ is the class of all smooth functions $u$ satisfying (25a). Moreover we shall restrict the competiting functions to be positive and monotonically decreasing. This is not a loss of generality, because the functional $J$ increases (and its domain is invariant) under the replacement $u(\cdot) \rightarrow$ $\rightarrow \int_{(\cdot)}^{+\infty}\left|u^{\prime}(t)\right| d t$

We subdivide the proof into several steps.

(i) Extremals. From the formula

$$
\frac{1}{J(u)} J^{\prime}(u)(v)=\frac{\int_{0}^{+\infty} r^{m-1}\left(|u|^{\mid \alpha-1} \operatorname{sgn} u\right) v d r}{\int_{0}^{+\infty} r^{m-1}|u|^{q} d r}-\frac{\int_{0}^{+\infty} r^{m-1}\left(\left|u^{\prime}\right|^{p-1} \operatorname{sgn} u^{\prime}\right) v^{\prime} d r}{\int_{0}^{+\infty} r^{m-1}\left|u^{\prime}\right|^{p} d r}
$$

for the Gateaux differential of the functional $J$, and the following consequence of the conditions $(25 a)$

$$
u(r)=o\left(r^{1-m^{\prime} / p}\right) \quad \text { as } r \rightarrow 0 \text { or }+\infty,
$$

we easily deduce, via Du Bois-Reymond lemma and integrations by parts, the following: the extremals of $J$ are solutions of differential equations of the form

$$
\left(r^{m-1}\left|u^{\prime}\right|^{p-1} \operatorname{sgn} u^{\prime}\right)^{\prime}+C r^{m-1}|u|^{q-1} \operatorname{sgn} u=0 \quad(C=\text { a positive constant })
$$

(2) Lemma 2 and its proof are closely related to a paper of BLIss [2]. 
verifying the conditions $(25 a, b)$ and

$$
u^{\prime}(r)=o\left(r^{-m / p}\right) \quad \text { as } r \rightarrow 0 \text { or }+\infty
$$

Conversely, every solution, endowed with the properties (25), of any differential equation of the form (28) is an extremal of $J$.

Fortunately, it is possible to represent in closed form a set of solutions, satisfying the conditions (25), of the differential equation (28). Note that if $p=2,(28)$ is a particularly simple case of Emden-Fowler equations and all its solutions can be obtained with quadratures. Indeed, let $p=2, m>2, q=2 m /(m-2)$, and let us consider only positive decreasing solutions. Putting $u(r)=r^{1-m / 2} v(r)$, we find for the new unknown the equation $r\left(r v^{\prime}\right)^{\prime}=(1-m / 2)^{2} v-C v^{q-1}$. This is equivalent to the first order equation $\left(r v^{\prime}\right)^{2}=(1-m / 2)^{2} v^{2}-(2 \sigma / q) v^{q}+$ constant, etc. Hence, when $p=2$, all the solutions of the equation (28) which are positive decreasing and have the properties (25) are of the form: $u(r)=\left(a+b r^{2}\right)^{1-m / 2}$ with $C=m(m-2) a b$. In the general case (i.e. $1<p<m, q=\ldots$ ) it is easy to check that

$$
\left(a+b r^{p^{\prime}}\right)^{1-m t_{p}}
$$

is a solution of (28), with the properties (25), when $a$ and $b$ are positive constants related to $O$ by the formula

$$
C=m\left(\frac{m-p}{p-1}\right)^{p-1} a b^{p-1} .
$$

Thus we have founded a two-parameter family of extremals of the functional $J$. In agreement with a previous remark, such extremals are positive decreasing functions. With a slight change in notation we represent these extemals in the form

$$
\varphi(r)=a\left(1+b r^{p^{\prime}}\right)^{1-m / p} \quad(a, b=\text { positive constants }) .
$$

The differential equation for the extremals in (29) becomes

$$
\left(r^{m-1}\left|\varphi^{\prime}(r)\right|^{p-1}\right)^{\prime}=m\left(\frac{m-p}{p-1}\right)^{p-1} a^{p-q} b^{p-1} r^{m-1} \varphi(r)^{q-1}
$$

(ii) An equivalent Lagrange problem. Our goal is to show that the extremals we have found in the previous subsection actually give the maximum. For this purpose it is convenient to put our problem

$$
J(u)=\text { maximum }
$$

24 - Annali di Matematica 
in the form of a Lagrange problem, namely:

$$
\begin{cases}\int_{0}^{+\infty} r^{m-1}\left|u_{1}(r)\right|^{q} d r=\text { maximum, } \\ \text { where } & \\ u_{2}^{\prime}(r)=r^{m-1}\left|u_{1}^{\prime}(r)\right|^{p} & \text { (a nonholonomic constraint) } \\ \text { and } & \\ u_{2}(0)=0, u_{1}(+\infty)=0, u_{2}(+\infty)=1 & \text { (boundary conditions). }\end{cases}
$$

Clearly the problem (31) is equivalent to the Lagrange problem (32). In fact, if $u$ is a solution of (31), then

$$
\left\{\begin{array}{l}
u_{1}(r)=u(r)\left(\int_{0}^{+\infty} t^{m-1}\left|u^{\prime}(t)\right|^{p} d t\right)^{-1 / p} \\
u_{2}(r)=\int_{0}^{r} t^{m-1}\left|u_{1}^{\prime}(t)\right|^{p} d t
\end{array}\right.
$$

is a solution of (32). Conversely, if a pair $\left(u_{1}, u_{2}\right)$ is a solution of (32), then $u=u_{1}$ is a solution of (31) because of the homogeneity of the functional $J$.

A set of extremals of the Lagrange problem is easily obtained from the results of the previous subsection; namely:

$$
\left\{\begin{array}{l}
\varphi_{1}(r)=a\left(1+b r^{p^{\prime}}\right)^{1-m / p} \quad(a, b=\text { positive constants }) \\
\varphi_{2}(r)=\int_{0}^{r} t^{m-1}\left|\varphi_{1}^{\prime}(t)\right|^{p} d t
\end{array}\right.
$$

More explicitly

$$
\varphi_{2}(r)=r^{m-p} \varphi_{1}(r)^{p} f\left(\frac{b r^{p^{\prime}}}{1+b r^{p^{\prime}}}\right)
$$

where $f(\xi)$ is the function

$$
f(\xi)=\frac{1}{p^{\prime}}\left(\frac{m-p}{p-1}\right)^{p} \xi^{p} \int_{0}^{1}(1-t)^{m / p^{\prime}}(1-\xi t)^{-m} d t .
$$

Claim: the two-parameter family of extremals (33) is a Mayer field in the first octant $\left\{\left(r, u_{1}, u_{2}\right) \in R^{3}: r>0, u_{1}>0, u_{2}>0\right\}$ of the three-dimensional euclidean space. In other words, the paths $] 0,+\infty\left[\ni r \rightarrow\left(r, \varphi_{1}(r), \varphi_{2}(r)\right)\right.$ are the trajectories of a smooth 
vector field $X$, defined in the first octant of $R^{3}$. Thus exactly one such path passes through any point of the first octant of $R^{3}$, and $X\left(r, u_{1}, u_{2}\right)$ is the slope at the point $\left(r, u_{1}, u_{2}\right)$ of the path passing through this point. The components of the vector field $X$ are given by

$$
\left\{\begin{array}{l}
X_{0}\left(r, u_{1}, u_{2}\right)=1 \\
X_{1}\left(r, u_{1}, u_{2}\right)=-\frac{m-p}{p-1}\left(u_{1} / r\right) \xi \\
X_{2}\left(r, u_{1}, u_{2}\right)=r^{m-1}\left|X_{1}\left(r, u_{1}, u_{2}\right)\right|^{p}
\end{array}\right.
$$

where $\xi$ is the root of the equation

$$
f(\xi)=r^{p-m} u_{1}^{-p} u_{2}, \quad 0<\xi<1 .
$$

The previous assertions are easily proven by solving the equations (33) with respect to $a$ and $b$. Note that the equations (35b) has exactly one solution $\xi$ for any righthand side as long as $r, u_{1}$ are positive and $u_{2} \geqslant 0$. In fact

$$
f(0)=0, \quad f(\xi) \rightarrow+\infty \text { if } \xi \rightarrow 1 \quad \text { and } \quad f^{\prime}(\xi)>0 \text { if } 0<\xi<1,
$$

for (34) gives

$$
\begin{cases}\xi^{-p} f(\xi) \rightarrow(m-p)^{p}(p-1)^{-p} /\left(m+p^{\prime}\right) & \text { if } \xi \rightarrow 0, \\ p^{\prime}\left(\frac{p-1}{m-p}\right)^{p}(1-\xi)^{-1+m / p} f(\xi)=\xi^{p-\left(m / p^{\prime}\right)-1} \int_{0}^{\xi} t^{m / p^{\prime}}(1-t)^{(m / 2)-2} d t & \\ \rightarrow B\left(-1+m / p, 1+m / p^{\prime}\right) & \text { if } \xi \rightarrow 1,\end{cases}
$$

and

$$
f^{\prime}(\xi)=\frac{1}{p^{\prime}}\left(\frac{m-p}{p-1}\right)^{p} \int_{0}^{1} \frac{(1-t)^{m / p^{\prime}}}{(1-\xi t)^{m+1}} \xi^{p-1}(p+(m-p) \xi t) d t .
$$

(iii) The Hilbert invariant integral. This is the main tool in our discussion of the Lagrange problem (32). We prove the following: There exists an exact differential $d W$ such that the integral $\int d W$, along any path $] 0,+\infty\left[\ni r \rightarrow\left(r, u_{1}(r), u_{2}(r)\right)\right.$ which satisfies the constraint $u_{2}^{\prime}(r)=r^{m-1}\left|u_{1}^{\prime}(r)\right|^{p}$, is $\geqslant \int_{0}^{+\infty} r^{m-1}\left|u_{1}(r)\right|^{a} d r$ and equality holds when the path is an extremal belonging to the Mayer field (33). To see this, we look tentatively at a twice continuously differentiable real valued function $W$ defined in the first octant of $R^{3}$ and enjoying the following property: For every point $\left(r, u_{1}, u_{2}\right)$ of the first octant of $R^{3}$, the (linear) function

$$
\left(\xi_{0}, \xi_{1}, \xi_{2}\right) \rightarrow r^{m-1} u_{1}^{q} \xi_{0}-\frac{\partial W}{\partial r}\left(r, u_{1}, u_{2}\right) \xi_{0}-\frac{\partial W}{\partial u_{1}}\left(r, u_{1}, u_{2}\right) \xi_{1}-\frac{\partial W}{\partial u_{2}}\left(r, u_{1}, u_{2}\right) \xi_{2}
$$


restricted to the cone of all directions issuing from the point $\left(r, u_{1}, u_{2}\right)$ such that

$$
\xi_{0}>0 \quad \text { and } \quad \xi_{0}^{p-1} \xi_{2}=r^{m-1}\left|\xi_{1}\right|^{p}
$$

has a critical point at $X\left(r, u_{1}, u_{2}\right)$. By critical point of a function, restricted to a manifold of codimension one, we mean a point in which the gradient of the function has a vanishing component parallel to the manifold. Note that, if the function is homogeneous and the manifold is a cone, the value of the function at any critical point is automatically zero.

From the Lagrange multipliers rule and the definition (35a) we obtain the relations

$$
\left\{\begin{array}{l}
\frac{\partial W}{\partial r}=r^{m-1} u_{1}^{g}+(p-1) r^{m-1}\left|X_{1}\right|^{p} \lambda \\
\frac{\partial W}{\partial u_{1}}=p r^{m-1}\left|X_{1}\right|^{p-1} \lambda \\
\frac{\partial W}{\partial u_{a}}=\lambda
\end{array}\right.
$$

where $\lambda$ is some continuously differentiable function to be determined. Incidentally, the system (39) implies that $\partial W / \partial X=r^{m-1} u_{1}^{\alpha}$ where

$$
\partial / \partial X=\partial / \partial r+X_{1} \partial / \partial u_{1}+r^{m-1}\left|X_{1}\right|^{p} \partial / \partial u_{2}
$$

is the derivative in the direction of the vector field $X$.

To find $\lambda$, we write down the compatibility conditions for the system (39). These compatibility conditions can be arranged in the form of an overdetermined system of linear partial differential equations of the first order in the unknown $\lambda$; namely

$$
\begin{aligned}
& \left(\begin{array}{ccc}
1 & 0 & -(p-1)\left(r^{m-1}\left|X_{1}\right|^{p}\right) \\
0 & 1 & -p\left(r^{m-1}\left|X_{1}\right|^{p-1}\right) \\
p\left(r^{m-1}\left|X_{1}\right|^{p-1}\right) & -(p-1)\left(r^{m-1}\left|X_{1}\right|^{p}\right) & 0
\end{array}\right) \times\left(\begin{array}{c}
\frac{\partial \lambda}{\partial r} \\
\frac{\partial \lambda}{\partial u_{1}} \\
\frac{\partial \lambda}{\partial u_{2}}
\end{array}\right)= \\
& q r^{m-1} u_{1}^{q-1}\left(\begin{array}{l}
0 \\
0 \\
1
\end{array}\right)-p \lambda\left(\begin{array}{c}
X_{1} \frac{\partial}{\partial u_{2}} \\
-\frac{\partial}{\partial u_{2}} \\
\frac{\partial}{\partial r}+X_{1} \frac{\partial}{\partial u_{1}}
\end{array}\right)\left(r^{m-1}\left|X_{1}\right|^{p-1}\right) \text {. }
\end{aligned}
$$


An analysis of the system (41) is very easy. In fact, since the matrix on the lefthand side of (41) has rank 2, we must impose orthogonality between the right-hand side of $(41)$ and the eigenvectors of the transposed matrix; i.e.

$$
p \lambda \frac{\partial}{\partial X}\left(r^{m-1}\left|X_{1}\right|^{p-1}\right)=q r^{m-1} u_{1}^{q-1}
$$

This gives the only possible value of $\lambda$. A more explicit formula is

$$
\lambda\left(r, u_{1}, u_{2}\right)=\frac{(p-1)^{p-1}}{(m-p)^{p}} \frac{r^{p} u_{1}^{q-p}}{\xi^{p-1}(1-\xi)},
$$

where $\xi$ is the root of the equation $(35 b)$. The formula (42b) follows from (42a) because

$$
\frac{\partial}{\partial X}\left(r^{m-1}\left|X_{1}\right|^{p-1}\right)=m\left(\frac{m-p}{p-1}\right)^{p-1} r^{m-p-1} u_{1}^{p-1} \xi^{p-1}(1-\xi),
$$

where $\xi$ is as before.

The equation (43) is an easy consequence of the definition of $X$ (= the slope of the Mayer field (33)) and of the differential equation (30) for the extremals of the original functional $J$. We can also check equation (43) by a direct computation. In this case one uses the equations (35) and the following formula for the derivative of the function (34)

$$
\xi(1-\xi) f^{\prime}(\xi)=\frac{1}{p^{\prime}}\left(\frac{m-p}{p-1}\right)^{p} \xi^{p}+(m-p)\left(\xi-\frac{1}{p^{\prime}}\right) f(\xi) .
$$

We can prove that the function $\lambda$, defined by (42), is actually a solution to the system (41). As the matrix at the left-hand side of (41) has rank 2, it is enough to verify two equations only. Disregarding (41.3) and combining (41.1) and (41.2) we have to cheek the following pair

$$
\left\{\begin{array}{l}
\frac{\partial}{\partial X} \lambda=0 \\
\frac{\partial}{\partial u_{1}} \lambda=p \frac{\partial}{\partial u_{\mathbf{2}}}\left(r^{m-1}\left|X_{1}\right|^{p-1} \lambda\right) .
\end{array}\right.
$$

Equation (45.1) is trivial since its characteristic lines are precisely the members of our Mayer field and the function $\lambda$ as defined by (42) is constant along any path of this field. Indeed, we can write $(42 b)$ as

$$
\lambda\left(r, u_{1}, u_{2}\right)=(\text { const })\left[u_{1}(1-\xi)^{1-n / p}\right]^{\alpha-p}\left[\frac{\xi}{1-\xi} r^{-p^{\prime}}\right]^{1-p} .
$$


On the other hand, the pair of equations

$$
u_{1}(1-\xi)^{1-m / p}=\mathrm{const} \quad \frac{\xi}{1-\xi} r^{-p^{\prime}}=\mathrm{const}
$$

(where $\xi$ is the root of $(35 b)$ ) is an alternative representation of the extremals belonging to the Mayer field (33).

The equation (45.2) follows from straightforward calculations using equations (35) and (44).

The procedure just described gives us a smooth solution $W$ to the system (39) when we use $\lambda$ as defined by (42). Let us show that the differential $d W$ has the property stated at the beginning; this will follow from the fact that $\lambda$ is positive. The difference

$$
\begin{aligned}
& E\left(r, u_{1}, u_{2} ; \xi_{0}, \xi_{1}, \xi_{2}\right)= \\
& r^{m^{\prime-1}} u_{1}^{q} \xi_{0}-\frac{\partial W}{\partial r}\left(r, u_{1}, u_{2}\right) \xi_{0}-\frac{\partial W}{\partial u_{1}}\left(r, u_{1}, u_{2}\right) \xi_{1}-\frac{\partial W}{\partial u_{2}}\left(r, u_{1}, u_{2}\right) \xi_{2}
\end{aligned}
$$

which we have already considered, is essentially the Weierstrass excess function. If the direction $\left(\xi_{0}, \xi_{1}, \xi_{2}\right)$ is so restricted that

$$
\xi_{0}>0 \quad \text { and } \quad \xi_{0}^{p-1} \xi_{2}=r^{m-1}\left|\xi_{1}\right|^{p}
$$

then we have from (39) the equation

$$
\begin{aligned}
E\left(r, u_{1}, u_{2} ; \xi_{0}, \xi_{1}, \xi_{2}\right)=- & \xi_{0} r^{m-1} \lambda\left(r, u_{1}, u_{2}\right) \times \\
& \times\left\{\left.\left|\xi_{1}\right| \xi_{0}\right|^{p}+p\left|X_{1}\left(r, u_{1}, u_{2}\right)\right|^{p-1}\left(\xi_{1} \mid \xi_{0}\right)+(p-1)\left|X_{1}\left(r, u_{1}, u_{2}\right)\right|^{p}\right\} .
\end{aligned}
$$

Clearly the expression in brackets is always $\geqslant 0$ and vanishes if (and only if) the direction $\left(\xi_{0}, \xi_{1}, \xi_{2}\right)$ is parallel to $X\left(r, u_{1}, u_{2}\right)$. As the factor in front is $\leqslant 0$, we obtain the desired result.

An explicit representation formula for the function $W$ can be derived; we need this formula to determine the boundary behaviour of $W$. Putting the $\lambda$ of (42) into (39) gives

$$
\left\{\begin{array}{l}
\frac{\partial W}{\partial r}=\frac{r^{m-1} u_{1}^{q}}{1-\xi} \quad \frac{\partial W}{\partial u_{1}}=\frac{q}{m} \frac{r^{m} u_{1}^{q-1}}{1-\xi} \\
\frac{\partial W}{\partial u_{2}}=\frac{(p-1)^{p-1}}{(m-p)^{p}} \frac{r^{p} u_{1}^{q-p}}{\xi^{p-1}(1-\xi)}
\end{array}\right.
$$

By (48), (35b) and $(36 b)$, the limit $W\left(r, u_{1}, 0+\right)$ exists at every boundary point $\left(r, u_{1}, 0\right)$ such that $r \neq 0$ and $u_{1} \neq 0$. In fact $W\left(r, u_{1}, 0+\right)=\left(r^{m} / m\right) u_{1}^{q}+$ constant. 
Thus

$$
\begin{aligned}
W\left(r, u_{1}, u_{2}\right)=W\left(r, u_{1}, 0+\right)+\int_{0}^{u_{z}} & \frac{\partial W}{\partial u_{2}}\left(r, u_{1}, u_{2}\right) d u_{2}= \\
& \text { Constant }+\left(r^{m} / m\right) u_{1}^{q}+\frac{(p-1)^{p-1}}{(m-p)^{p}} r^{m} u_{1}^{q} \int_{0}^{\xi} \frac{f^{\prime}(\xi) d \xi}{\xi^{p-1}(1-\xi)},
\end{aligned}
$$

after having performed an obvious change of variables in the integral. An integration by parts and equation (44) give

$$
(q / p) \int_{0}^{\xi} \frac{f^{\prime}(\xi) d \xi}{\xi^{p-1}(1-\xi)}=\frac{f(\xi)}{\xi^{p-1}(1-\xi)}+\left(\frac{m-p}{p-1}\right)^{p-1} \frac{\xi}{1-\xi} .
$$

Therefore

$$
W\left(r, u_{1}, u_{2}\right)=C+\frac{r^{m} u_{1}^{q}}{m(1-\xi)}+\left(\frac{p-1}{m-p}\right)^{p-1} \frac{r^{p} u_{1}^{q-p} u_{2}}{m \xi^{p-1}(1-\xi)}
$$

where $C$ is a constant and $\xi$, as always, is the root of equation (35b).

(iv) Oonclusion. The concluding argument is standard. Let $\left(u_{1}, u_{2}\right)$ be a test pair for the Lagrange problem (32), namely

$$
\left\{\begin{array}{l}
\int_{0}^{+\infty} r^{m-1}\left|u_{1}^{\prime}(r)\right|^{p} d r=1, \quad u_{1}(r) \rightarrow 0 \text { as } r \rightarrow+\infty, \\
u_{2}(r)=\int_{0}^{r} t^{m-1}\left|u_{1}^{\prime}(t)\right|^{p} d t .
\end{array}\right.
$$

According to a remark made at the beginning we can suppose that $u_{1}$ is positive and decreasing; of course $u_{2}$ is positive and increasing so the path $] 0,+\infty[\ni r \rightarrow$ $\rightarrow\left(r, u_{1}(r), u_{2}(r)\right)$ lies in the first octant of $R^{3}$.

From the representation formula (49), the asymptotic behaviours $(36 b)$ and

$$
u_{1}(r)=o\left(r^{1-m / p}\right) \quad \text { as } r \rightarrow 0 \text { or }+\infty,
$$

which is a consequence of (50.1), we easily conclude that

$$
W\left(r, u_{1}(r), u_{2}(r)\right) \rightarrow C \quad \text { as } r \rightarrow 0
$$

Also $W(+\infty, 0,1)$, the limit of $W\left(r, u_{1}(r), u_{2}(r)\right)$ as $r \rightarrow+\infty$, exists and is independent of the path. From $(36 b),(49)$ and (51) we get

$$
W(+\infty, 0,1)=C+m^{-q / p}\left[\frac{p-1}{m-p}\right]^{q / p^{\prime}}\left[\frac{1}{p^{\prime}} B\left(m / p, m / p^{\prime}\right)\right]^{-q / m} \text {. }
$$


Therefore, from subsection (iii),

$$
\int_{0}^{+\infty} r^{m-1}\left|u_{1}(r)\right|^{q} d r \leqslant W(+\infty, 0,1)-O
$$

and the equality holds if (and only if) the pair $\left(u_{1}, u_{2}\right)$ belong to the Mayer field (33).

This concludes the proof.

\section{3. - Proof of the theorem $=$ lemma $1+$ lemma 2 .}

\section{REFERENCES}

[1] L. Bers - F. JohN - M. SChechter, Partial Differential Equations, Interscience (1964)

[2] G. A. Burss, An integral inequality, Journal London Math. Soc., 5 (1930).

[3] H. FEDERER, Curvature measure, Trans. Amer. Math. Soc., 93 (1959).

[4] H. Federer - W. Fuming, Normal and integral currents, Annals of Math., 72 (1960).

[5] W. Fleming, Functions whose partial derivatives are measures, Illinois J. Math., 4 (1960).

[6] W. Fumming - R. Rishel, An integral formula for total gradient variation, Arch. Math., II (1960).

[7] M. Mrranda, Distribuzioni aventi derivate misure, Ann. Scuola Norm. Sup. Pisa, 18 (1964).

[8] M. Mrranda, Sul minimo dell'integrale del gradiente di una funzione, Ann. Scuola Norm. Sup. Pisa, 19 (1965).

[9] M. Mrranda, Disuguaglianze di Sobolev sulle ipersuperfioi minimali, Rend. Sem. Mat. Univ. Padova, 38 (1967).

[10] G. Rosen, Minimum value for $c$ in the Sobolev inequality, STAM J. Appl. Math., 21 (1971).

[11] S. L. SовоLev, On a theorem of functional analysis (in russian), Mat. Sb., 4 (1938),

[12] S. L. SoBoLEV, Applications of functional analysis in mathematical physics, Amer. Math. Soc. $(1963)$.

[13] L. C. Young, Partial area, Rivista Mat. Univ. Parma, 10 (1959). 